\title{
Measurements of a Newly Designed BPM for the Tevatron Electron Lens 2
}

\author{
V. E. Scarpine, B. Fellenz, G. Kuznetsov, V. Kamerdzhiev, M. Olson, V. \\ D. Shiltsev and X. L. Zhang
}

Fermi National Accelerator Laboratory, P.O. Box 500, Batavia, IL 60510, USA

\begin{abstract}
Fermilab has developed a second electron lens (TEL-2) for beam-beam compensation in the Tevatron as part of its Run II upgrade program. Operation of the beam position monitors (BPMs) in the first electron lens (TEL-1) showed a systematic transverse position difference between short proton bunches ( $2 \mathrm{~ns}$ sigma) and long electron pulses ( 1 us) of up to $\sim 1.5 \mathrm{~mm}$. This difference was attributed to frequency dependence in the BPM system. The TEL-2 BPMs utilize a new compact four plate design with grounding strips between plates to minimize crosstalk. In-situ measurements of these new BPMs are made using a stretched wire pulsed with both proton and electron beam formats. In addition, longitudinal impedance measurements of the TEL-2 are presented. Signal processing algorithm studies indicate that the frequency dependent transverse position offset may be reduced to $\sim 0.1 \mathrm{~mm}$ for the beam structures of interest.
\end{abstract}

Keywords: BPM

PACS: 07.77.Ka 29.27.-a 29.27.Fh

\section{INTRODUCTION}

The Tevatron has operated an Electron Lens (TEL-1) mainly as a gentle remover of DC beam in the abort gaps [1]. This DC beam component, if not removed, is enough to cause a quench during an abort or possibly damage silicon detectors in the CDF and D0 experiments. In addition, the TEL-1 has been used as a R\&D project for Tevatron beam-beam compensation [2]. Based on the success of this project, a second Tevatron Electron Lens (TEL-2) was designed and constructed [3]. Operation of two electron lenses will allow for simultaneous vertical and horizontal beam-beam compensation as well as a spare DC beam remover. Development of the TEL-2 allowed for upgrades from the initial TEL-1 design, including a new beam position monitor (BPM) design [4].

Accurate positioning of the electron beam relative to the Tevatron proton or antiproton beam is critical in order to have efficient DC beam removal and beam-beam compensation. Figure 1(a) shows a schematic of the TEL-1, including the location of four diagonal split-plate BPMs used to measure electron, proton and antiproton transverse beam positions. Operation of the electron guns in the electron lenses limits the electron pulse width to greater than $\sim 1 \mu$ s, while the proton and antiproton bunches are a few ns in length. Figure 1(b) is a plot of stretched-wire measurements of beam position versus signal frequencies from 20 to 100 ns, showing a clear frequency dependent position. 


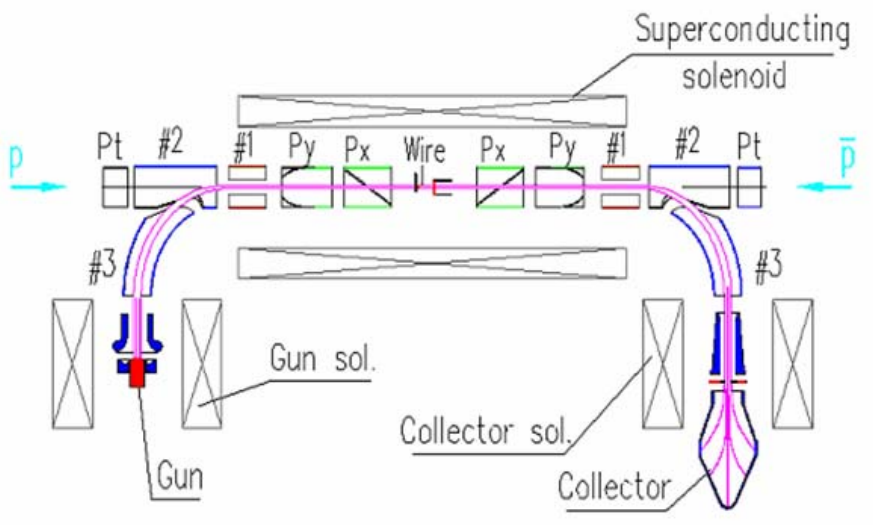

(a)

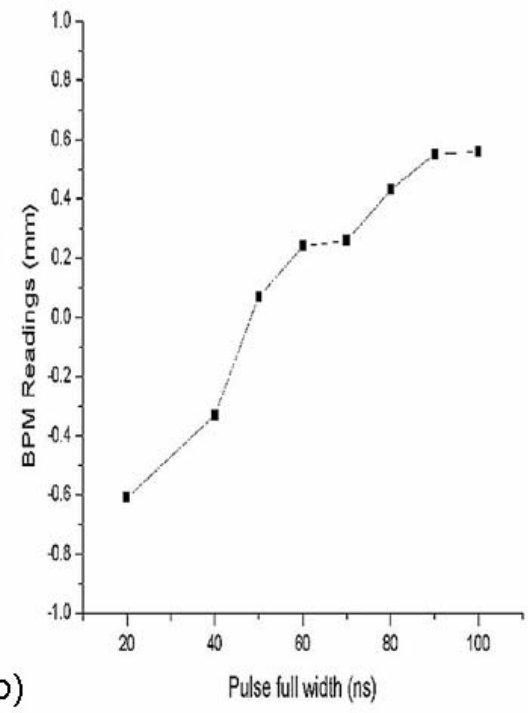

FIGURE 1. (a) Schematic layout of the TEL-1. (b) TEL-1 BPM position versus beam pulse length.

\section{TEL-2 BEAM POSITION MONITORS}

While diagonal split-plate BPMs give good linearity, they require two sets of pickups to measure horizontal and vertical positions. For TEL operations, it is necessary to measure beam positions very precisely. Figure 2 shows a new compact four-plate BPM design that is utilized in the TEL-2. This design is compact and has ground strips between pickups to minimize signal crosstalk [4].

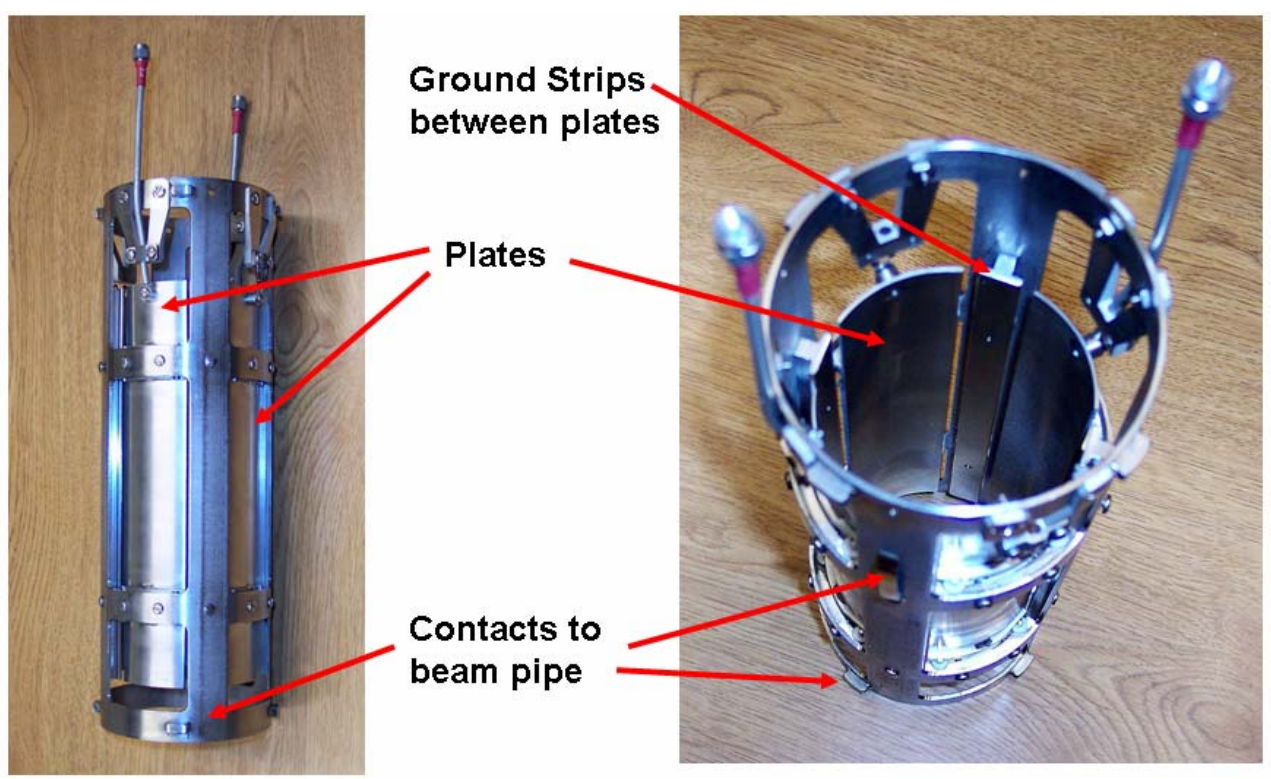

FIGURE 2. New four-plate BPM design for the TEL-2. 


\section{TEL-2 Stretch-Wire BPM Measurements}

The TEL-2 BPMs were calibrated in-situ. A wire was stretched through the entire TEL-2 system and pulsed while BPM signals were measured. Figure 3(a) shows a single BPM plate response versus frequency. There are a number of potential resonance frequencies. Figure 3(b) gives the measured impedance versus frequency and shows a number of impedance spikes. These impedance spikes are likely due to ground path structures in the TEL-2 systems causing resonance effects.

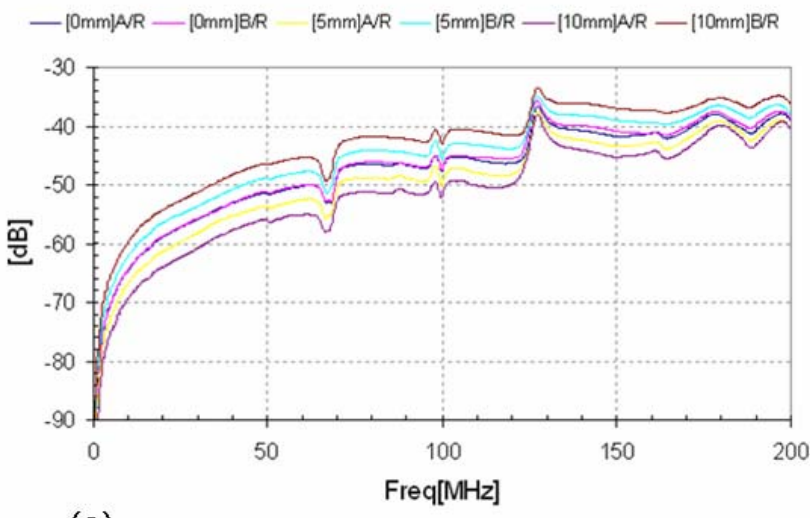

(a)

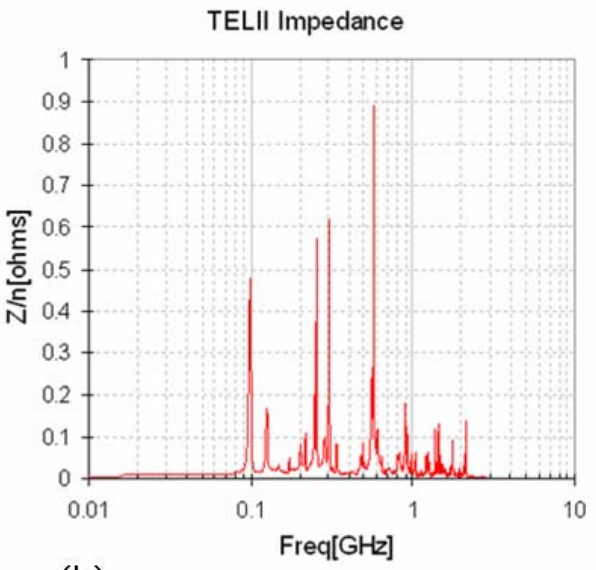

(b)

FIGURE 3. (a) Stretched-wire single BPM plate response versus frequency for new TEL-2 BPM design. (b) Measured TEL-2 impedance from stretched wire.

Figure 4 plots the BPM measured wire position versus frequency for different horizontal stretched-wire locations. One can see clearly that the BPM measured wire position is dependent on the frequency of the pulsed signal and can have dramatic changes for frequencies above $100 \mathrm{MHz}$.

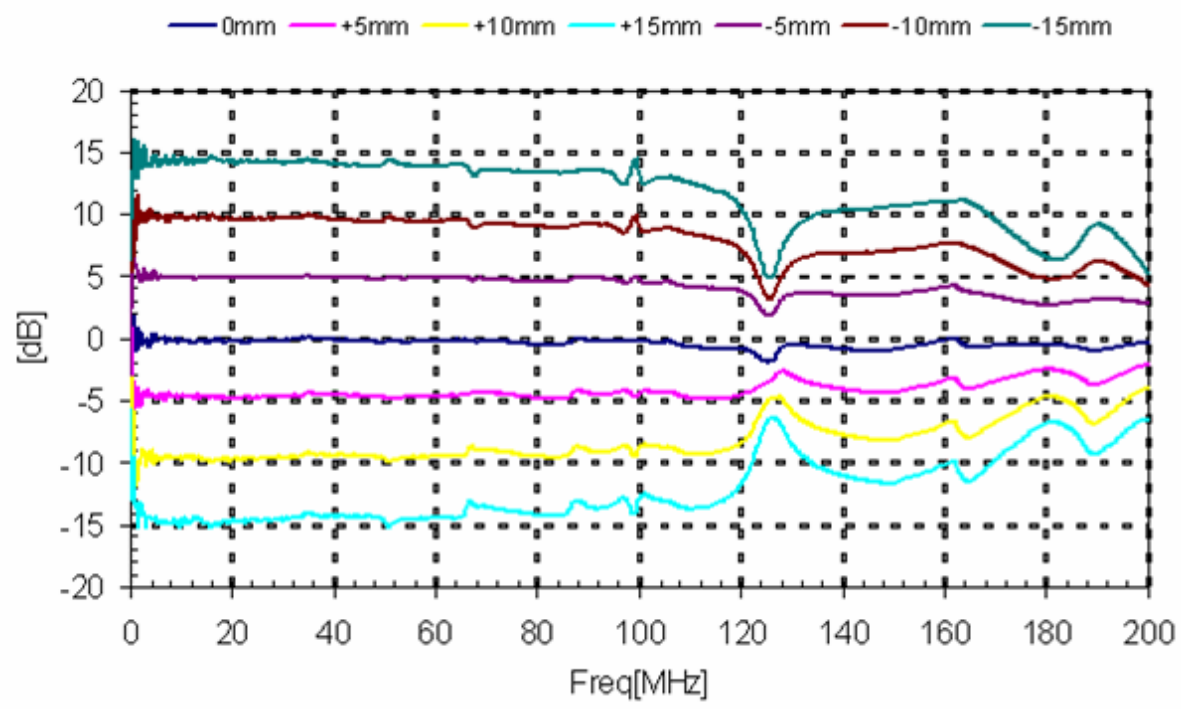

FIGURE 4. BPM measured position versus frequency for different stretched wire positions. 
BPM signal processing for the TEL-1 was based on time-domain processing and integrated over the entire BPM signal frequency. Figure 4 show that integrating over the entire frequency range will yield incorrect beam position measurements. Stretchedwire measurements were made of the TEL-2 BPMs for electron-beam-type signals of $\sim 1 \mu$ s pulses and proton-beam-type signals of $\sim 2$ ns $\sigma$ gaussian pulses. BPM plate signals were acquired with an oscilloscope and processed offline. The wide frequency band processing uses the peak value of a cumulative integral of the BPM plate signals. The difference divided by the sum of these peak values gives the measured beam position in normalized units. Figure 5(a) shows a TEL-2 electron BPM plate signal and its cumulative integral plot, while Fig. 5(b) shows the same for a proton BPM plate signal. Figure 6 shows a plot of the difference between the measured electron and proton positions for different horizontal stretched-wire positions for TEL-2 upstream and downstream, vertical and horizontal BPMs.
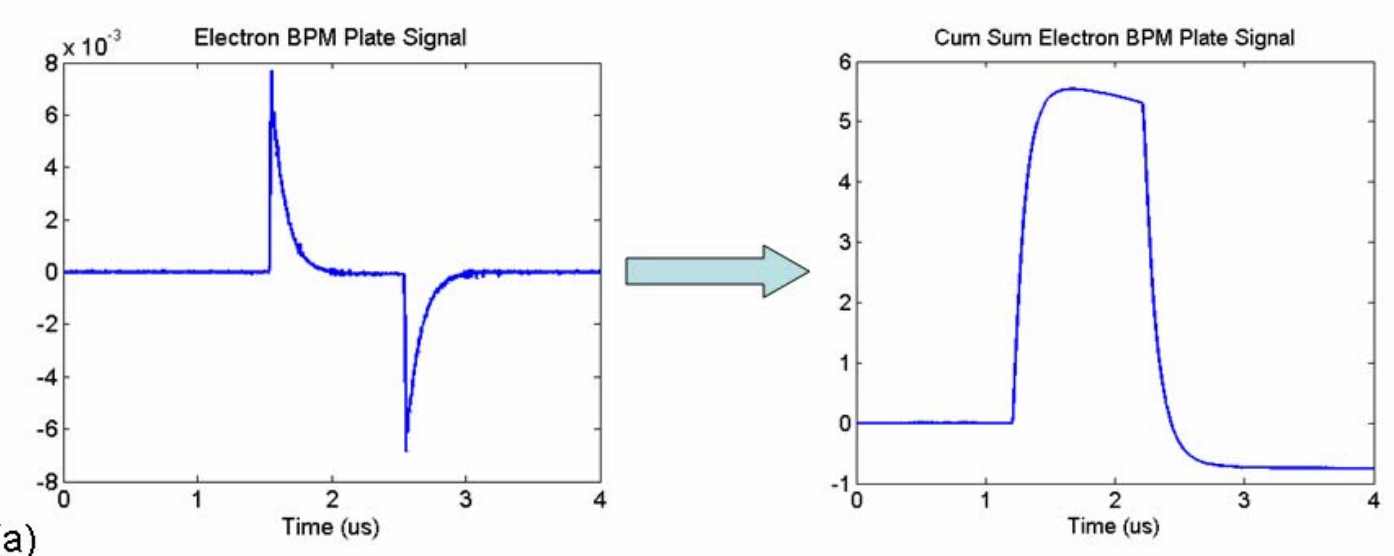

(a)
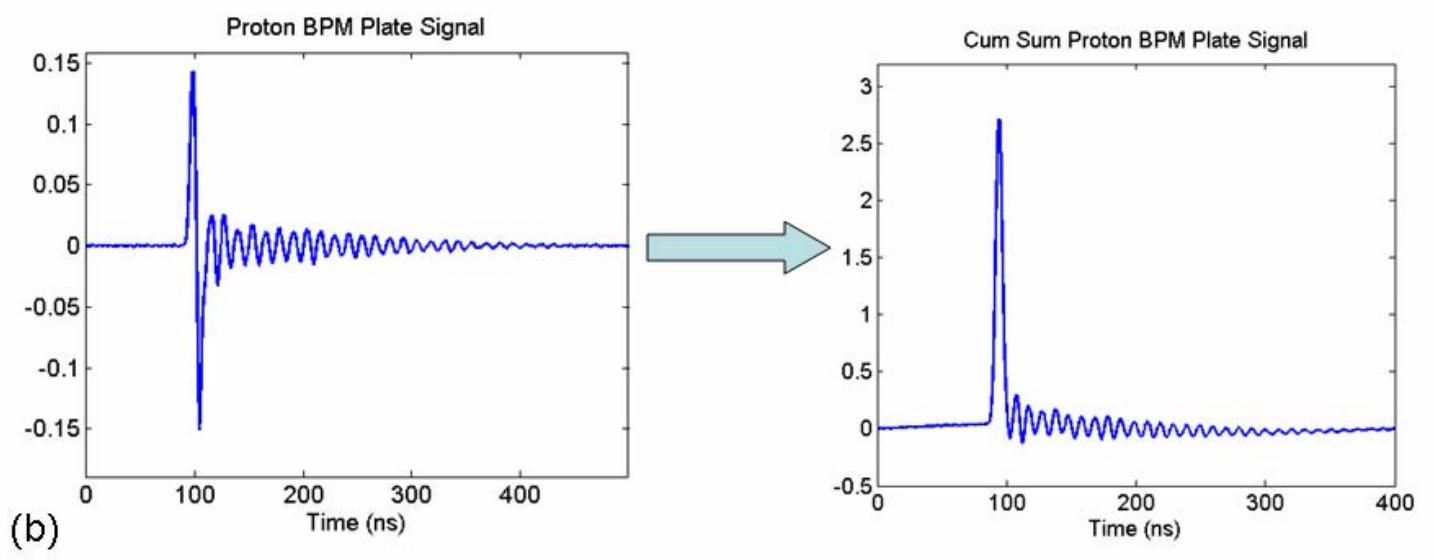

FIGURE 5. (a) Electron BPM plate signal and its cumulative integral plot. (b) Proton BPM plate signal and its cumulative integral plot. 


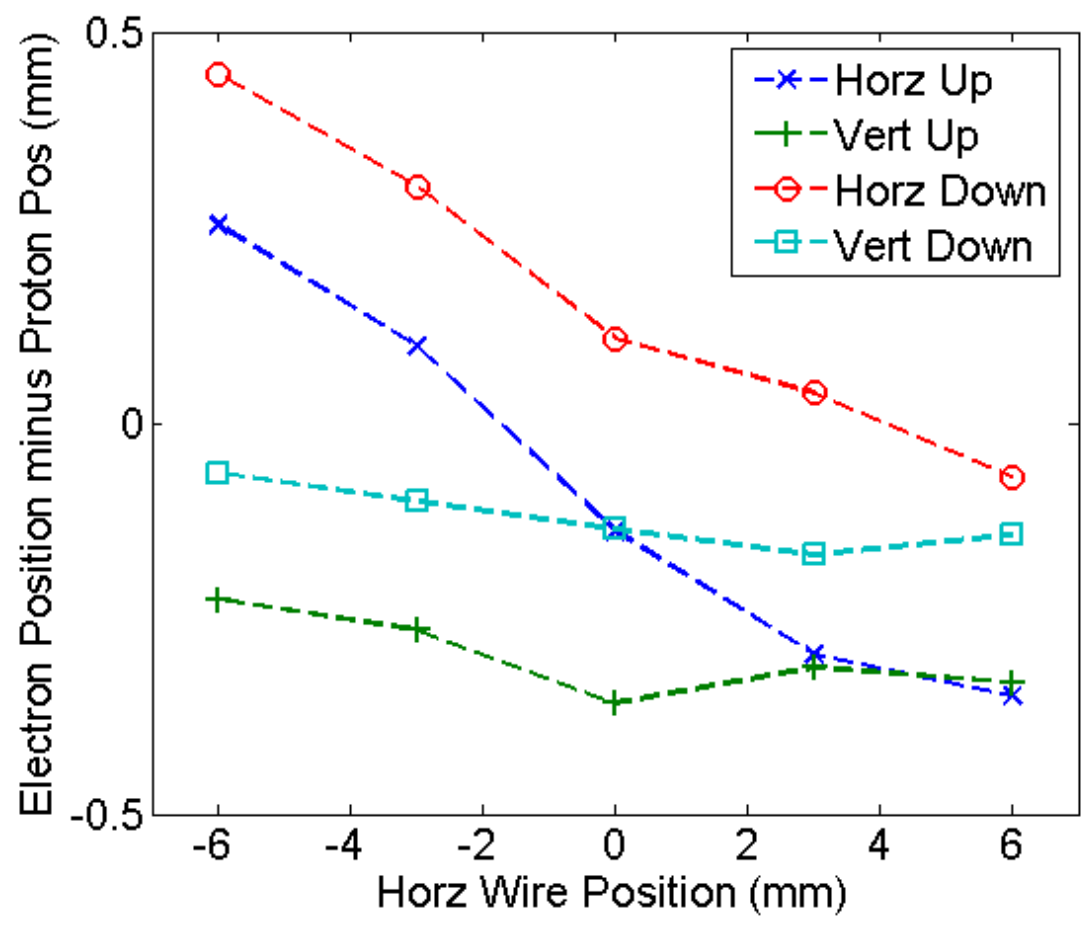

FIGURE 6. TEL-2 BPM difference between the measured electron and proton positions for different horizontal stretched-wire positions using a wide frequency band algorithm. Different curves are for upstream and downstream, vertical and horizontal BPMs.

\section{Narrow Frequency Band Position Measurements}

Electron and proton position differences are attributed to the signal processing over a wide frequency range. The same electron and proton BPM signals acquired for the wide frequency band signal processing are now reprocessed offline over a narrow frequency range. Figure 7(a) shows a TEL-2 electron BPM plate signal and its fast frequency response (FFT), while fig. 7(b) shows the same for a proton BPM plate signal. Since the electron and proton spectrums are so different, a compromise choice of narrow frequency band is needed. For our processing we choose the band of 5 to 20 $\mathrm{MHz}$, which gives good overlap for both spectrums. The electron and proton spectrums processed with a 15-MHz-wide Hanning window centered at $12.5 \mathrm{MHz}$. The output of the Hanning window is integrated to give a single value for the BPM plate. The difference divided by the sum of these peak values gives the measured beam position in normalized units. Figure 8 shows a plot of the difference between the measured electron and proton positions for different horizontal stretched-wire positions for TEL-2 upstream and downstream, vertical and horizontal BPMs. These data shows improved BPM measurements over the wide frequency band data shown in Fig. 6. 

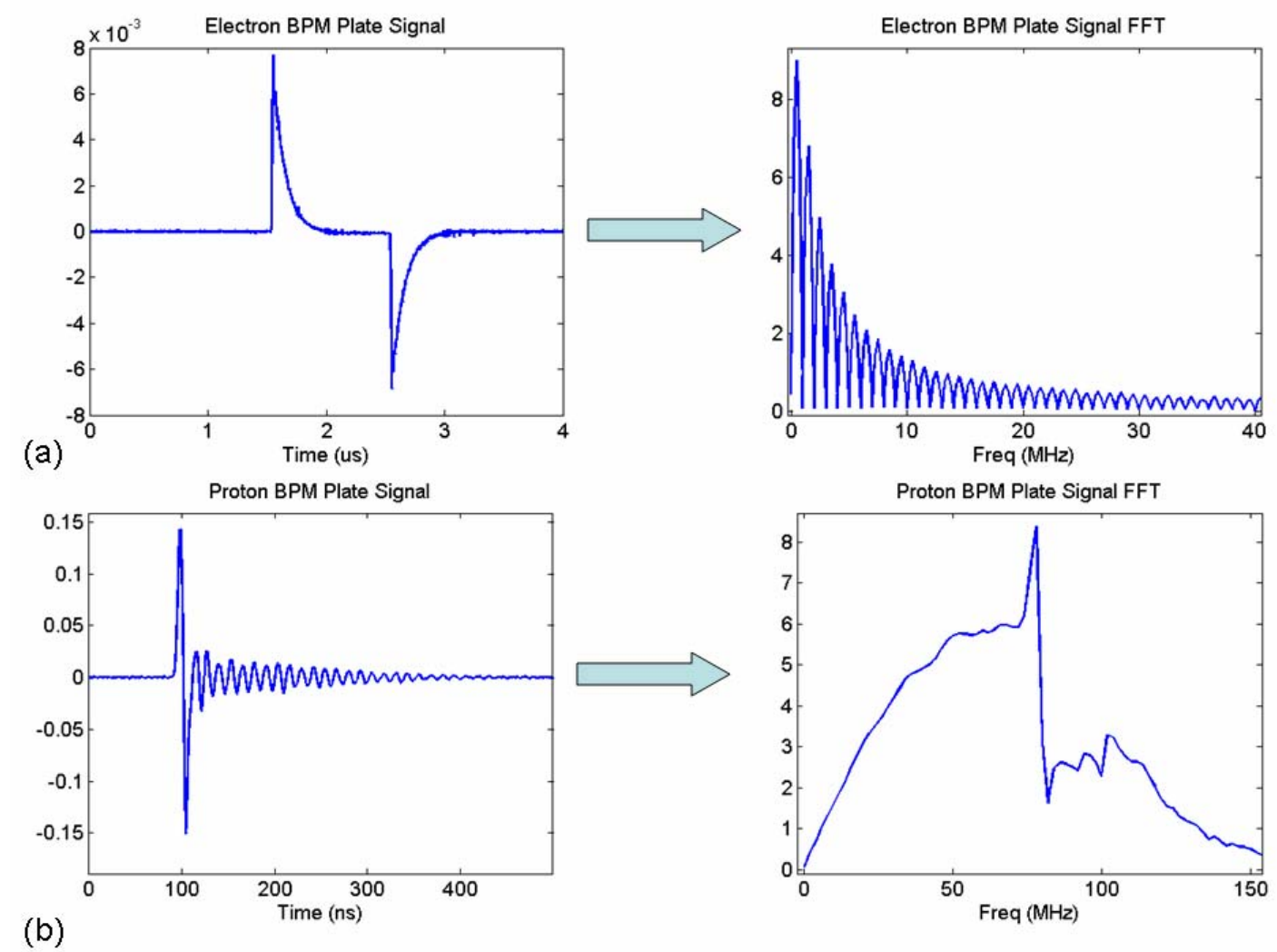

FIGURE 7. (a) Electron BPM plate signal and FFT plot. (b) Proton BPM plate signal and its FFT plot.

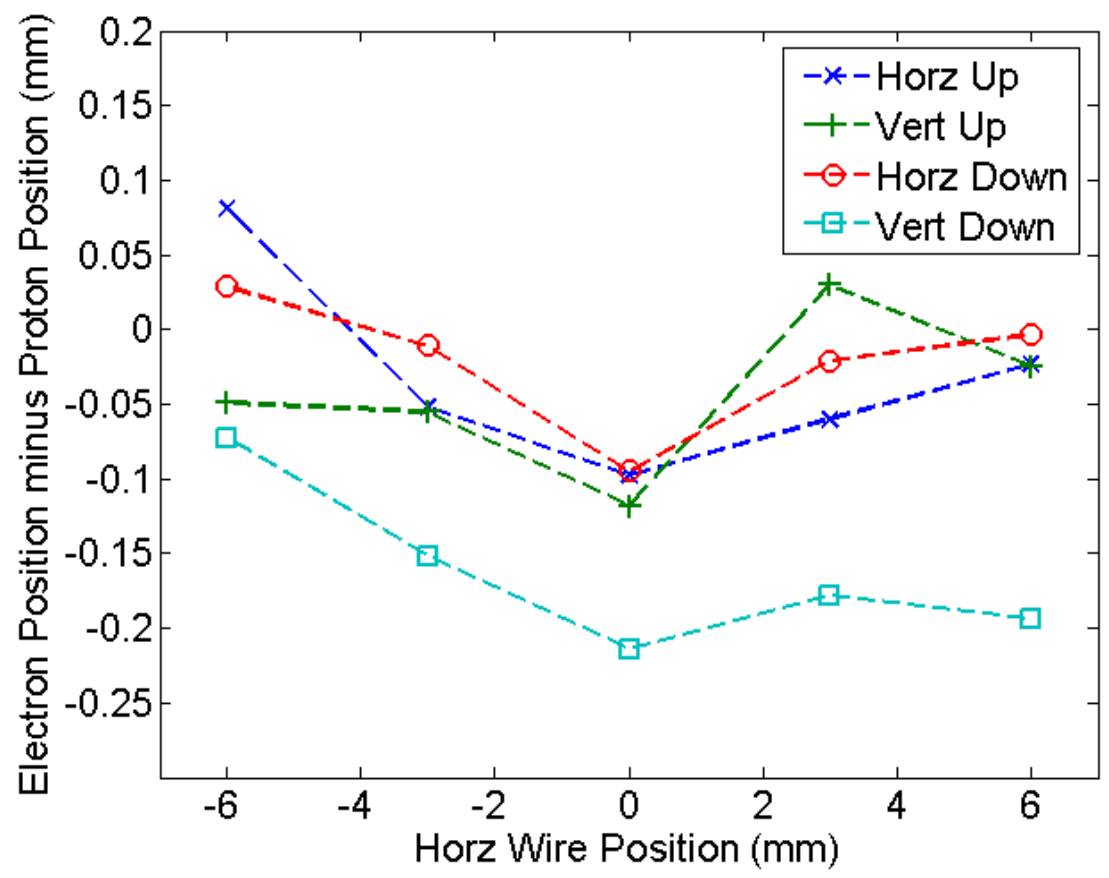

FIGURE 8. TEL-2 BPM difference between the measured electron and proton positions for different horizontal stretched-wire positions using a narrow frequency band algorithm. Different curves are for upstream and downstream, vertical and horizontal BPMs. 


\section{CONCLUSION}

A compact four-plate BPM design has been tested for the new TEL-2. Measurements from the TEL-1 and TEL-2 BPMs show that there is frequency dependence to the measured beam positions. Development of a narrow frequency band algorithm reduces the measured beam position difference to level which is acceptable for DC beam removal and beam-beam compensation.

\section{ACKNOWLEDGMENTS}

We thank our colleagues Howie Pfeffer, Bob Webber, Manfred Wendt, Jim Crisp, Greg Saewert and Jim Fitzgerald. This work was supported by the U.S. Department of Energy under contract No. DE-AC02-76CH03000.

\section{REFERENCES}

1. X. Zhang, V. Shiltsev, F. Zimmerman and K. Bishofberger, "The Special Applications of the Tevatron Electron Lens in Collider Operations," presented at the Particle Accelerator Conference, Knoxville, Tennessee, 2003.

2. K. Bishofberger, G. Kuznetsov, V. Shiltsev, X. Zhang, "Tune-Shift Compensation Using the Electron Lens," presented at the Particle Accelerator Conference, Knoxville, Tennessee, 2003.

3. V. Shiltsev, X. L. Zhang, G. Kuznetsov, H. Pfeffer, G. Saewert, D. Wolff, R. Hively, V. Kamerdzhiev, A. Martinez, A. Klebaner, K. Bishofberger, F. Zimmerman, M. Tiunov, A. Kuzmin, I. Bogdanov, E. Kashtanov, S. Kozub, V. Sytnik and L. Tkachenko, "Tevatron Beam-Beam Compensation Project Progress," presented at the Particle Accelerator Conference, Knoxville, Tennessee, 2005.

4. X. Zhang, J. Crisp, J. Fitzgerald, G. Kuznetsov, M. Olson, H. Pfeffer, G. Saewert, A. Semenov, V. Shiltsev, N. Solyak, D. Wildman, K. Bishofberger, E. Kashtanov, S. Kozub, L. Tkachenko and V. Sytnik, "Upgrades of the Tevatron Electron Lens," presented at the Particle Accelerator Conference, Knoxville, Tennessee, 2003. 